\title{
Frontières
}

\section{Bibliographie indicative}

\section{Lise Lalonde}

Volume 14, numéro 1, automne 2001

URI : https://id.erudit.org/iderudit/1074175ar

DOI : https://doi.org/10.7202/1074175ar

Aller au sommaire du numéro

Éditeur(s)

Université du Québec à Montréal

ISSN

1180-3479 (imprimé)

1916-0976 (numérique)

Découvrir la revue

Citer ce document

Lalonde, L. (2001). Bibliographie indicative. Frontières, 14(1), 79-82.

https://doi.org/10.7202/1074175ar

Ce document est protégé par la loi sur le droit d'auteur. L'utilisation des services d'Érudit (y compris la reproduction) est assujettie à sa politique d'utilisation que vous pouvez consulter en ligne.

https://apropos.erudit.org/fr/usagers/politique-dutilisation/
Cet article est diffusé et préservé par Érudit.

Érudit est un consortium interuniversitaire sans but lucratif composé de l’Université de Montréal, l'Université Laval et l'Université du Québec à Montréal. Il a pour mission la promotion et la valorisation de la recherche. https://www.erudit.org/fr/ 
l'Europe, coll. «Questions Actualité », $500 \mathrm{p}$.

\section{HISTOIRE / RÉCITS D'EXIL EN MARGE DU POUVOIR}

CUBERO, José (1998). Histoire du vagabondage du Moyen Âge à nos jours, Paris, Éditions Imago, 294 p.

DE LAS CASES, Emmanuel (1999). Mémorial de Sainte-Hélène (Tomes 1 et 2), Paris, Éditions du Seuil, coll. «Point».

LABERGE, Danielle (1997). Marginaux et marginalité. Les États-Unis aux XVIII e et XIXe siècles, Paris, L'Harmattan, coll. "Logiques sociales", $279 p$.

\section{INTERDISCIPLINARITÉ / EN MARGE DES DISCIPLINES}

\section{BIBLIOGRAPHIE} INDICATIVE

Lise Lalonde

\section{DROIT}

BERTRAND, Marie-Andrée (dir.) (1996). Numéro spécial de la Revue canadienne Droit et Société, Thématique "Le droit comme instrument d'exclusion ", vol. 11, n² (automne). ROBERT, Pierre (dir.) (1998). La gestion sociale par le droit pénal: la discipline du travail et la punition des pauvres. Actes de la 8 e Journée de droit social et du travail (Journée tenue en 1997 à l'Université du Québec à Montréal), Cowansville, Éditions Y. Blais, $171 \mathrm{p}$.

\section{SANTÉ, MALADIE ET MORT}

CROMBEZ, Jean-Charles (1998). La personne en écho: cheminements dans la complexité, Beauport, MNH, $206 \mathrm{p}$.

CROMBEZ, Jean-Charles (1994). La guérison en écho, Beauport, MNH, $448 \mathrm{p}$.

FLIS-TREVES, Muriel (2001). Ce savoir nouveau sur la vie et la mort, Paris, Plon, $150 \mathrm{p}$

GLANNON, Walter (2000). "Tracing the soul: Medical decisions at the margins of life ", Christian Bioethics, vol. 6, n० 1, p. 49-69.

Réseau européen de coopération scientifique et al. (1997). Médecine et droits de l'homme. La santé face aux droits de l'homme, à l'éthique et aux morales: cent vingt cas examinés sous l'angle des normes juridiques, des repères éthiques internationaux et européens, des morales catholique, protestante, juive, musulmane, bouddhiste et agnostique comme outil d'aide à la décision et à la pédagogique, Éditions du Conseil de

BOUVRIER, Pierre (2000). « Le disciplinaire et l'interdisciplinaire " ordinaire " ", Sociologie et Sociétés, vol. XXXII, $n^{\circ} 1$ (printemps), p. 229-234.

DUCHASTEL, Jules et Danielle LABERGE (1999). " La recherche comme espace de médiation interdisciplinaire", Sociologie et Sociétés, vol. XXXI, $\mathrm{n}^{\circ} 1$ (printemps), p. 63-76.

DUMAS, Brigitte (1999). "Les savoirs nomades ", Sociologie et Sociétés, vol. XXXI, $n^{\circ} 1$ (printemps), p. 51-62. GLOBE, REVUE INTERNATIONALE D'ÉTUDES QUÉBÉCOISES (2001). Diverses disciplines et perspectives critiques portent leur regard sur le thème " Les marges de l'identité au Québec ». L'appel de textes propose: "L'identité québécoise, prise entre une dynamique mondiale marquée par l'intégration, l'accélération, le mouvement et le métissage, inscrite au cœur d'une impasse constitutionnelle dont la résolution, à court et à moyen terme, n'est guère envisageable, permet des interprétations plurielles et éclatées ». Montréal, Université McGill (automne, à paraître).

\section{LITTÉRATURE ET MARGE /} LITTÉRATURE À LA MARGE

ACQUELIN, José (1994). Cité ouverte: itinérance et marginalité, Montréal, Les Productions Cité ouverte 2002, 64 p.

BIRON, Michel et Pierre POPOVIC (1998). Un livre dont vous êtes l'intellectuel, Saint-Laurent, Québec, Fidès, $192 \mathrm{p}$.

BORGES, Jorge Luis (1967). L'Aleph, Paris, Gallimard, coll. « L'Imaginaire », $218 p$.

BORGES, Jorge Luis (1965). Fictions, Paris, Gallimard, coll. «Folio », 209 p.

CÉLINE, Louis-Ferdinand (1996). Voyage au bout de la nuit, Paris, Gallimard, coll. « Folio Plus », 558 p.
CHEVRIER, Jacques (1995). William Sassine: écrivain de la marginalité, Toronto, Éditions du Gref, coll. « L'Un pour l'autre ", $335 \mathrm{p}$.

DE BEAUVOIR, Simone (1981). La cérémonie des adieux, suivi de Entretiens avec Jean-Paul Sartre. Août-Septembre 1974, Paris, Gallimard, $559 \mathrm{p}$.

ECO, Umberto (1982). Le nom de la rose, Paris, Grasset, $633 \mathrm{p}$.

FALZONI, F.M. (dir.) (1987). La deriva delle francofonie (L'altérité dans la littérature québécoise), premier séminaire annuel consacré aux littératures francophones (dont celui des 22-23 octobre 1986 dédié à la littérature québécoise), Centro Studi Sorelle Clarke Dell' Università di Bologna, Bologna, Cooperativa Libraria Universitaria Editrice Bologna (CLUEB), $281 \mathrm{p}$.

HAREL, Simon (1999). Le voleur de parcours. Identité et cosmopolitisme dans la littérature québécoise contemporaine: essai, Montréal, XYZ Éditeur, coll. « Documents », 334 p.

MAILLET, Antonine (1990). Pélagiela-charrette, Montréal, Bibliothèque québécoise, $334 \mathrm{p}$.

OPEN CITY PRODUCTIONS 2002, Homelessness and Marginalization, Compilation of poems and short stories donated by some of Quebec's most well-known authors on the themes of isolation, exclusion and discrimination, Montreal. (Depuis 1995, des milliers de copies de cet ouvrage ont été distribuées gratuitement aux écoles secondaires québécoises qui en font la demande. On peut communiquer avec cette compagnie à l'adresse électronique: info@opencity.ca).

ORWELL, George (1980). 1984, Paris, Gallimard, coll. « Folio », 438 p.

PEZECHKIAN-WEINBERG, Pary (1998). Michel Tournier : marginalité et création, New York, Peter Lang Publishing, $170 \mathrm{p}$.

PYNCHON, Thomas (1988). L'arc-enciel de la gravité, Paris, Seuil, coll. «Fiction \& Cie », $761 \mathrm{p}$.

SCANLAN, Margaret (2001). Plotting Terror: Novelist and Terrorists in Contemporary Fiction, Charlottesville, VA, University of Virginia Press, 224 p.

\section{PÉDAGOGIE}

BIET, Christian, Christine FÉRETFLEURY et Sylvie FLORIAN-POUILLOUX (dir.) (1996). Exclusion et marginalité ou L'art de témoigner de son temps dans la littérature et dans l'actualité, Paris, Gallimard Jeunesse, coll. « Les Thématiques ", $223 \mathrm{p}$.

GIROUX, Henry A. (1997). Pedagogy and the Politics of Hope: Theory,
Culture, and Schooling, Boulder, CO, Westview Press, $290 \mathrm{p}$.

\section{PHILOSOPHIE CONTEMPORAINE ET MARGES}

BAUDRILLARD, Jean (1999). Le paroxyste indifférent, Paris, Librairie générale française, coll. "Biblio", $184 \mathrm{p}$.

FOUCAULT, Michel, Les anormaux: cours au Collège de France (19741975), édition établie en 1999 sous la direction de F. EWALD, A. FONTANA, V. MARCHETTI et A. SALOMONI, Paris, Gallimard, $351 \mathrm{p}$.

\section{SCIENCES HUMAINES, SOCIALES ET ÉCONOMIQUES}

ARNOLDO, Carlos A. (dir.) (2001). Child Abuse on the Internet. Ending the Silence, UNESCO Publishing/ Berghahn Books, $220 \mathrm{p}$.
AUTES, Michel (1992). "Socialité et marginalité ", Travail social et pauvreté, Paris, Syros - Alternatives, p. 173-189.

BADINTER, Robert (2000). L'abolition, Paris, Fayard, $400 \mathrm{p}$.

BAKER, Patrick L. (1994). Centering the Periphery. Chaos, Order, and the Ethnohistory of Dominica, Montréal, McGill-Queen's University Press, 328 p.

BAMMATE, Najm-oud Dine (2000). L'Islam et l'Occident. Dialogues, Préface de Jean d'Ormesson, Éditions UNESCO/Éditions Christian Destremau, $168 \mathrm{p}$.

BARBER, Benjamin R. (1996). Djihad Brouwer, coll. "Sociologie économique », $304 \mathrm{p}$.

BAREL, Yves (1984). La société du vide, Paris, Seuil, $267 \mathrm{p}$.

BAREL, Yves (1982). La marginalité sociale, Paris, Presses universitaires de France, $250 \mathrm{p}$.

BAUMAN, Zygmunt (1999). Le coût humain de la mondialisation, Paris, Hachette, coll. « Forum », 204 p.

BECKER, Howard S. (1985). Outsiders. Étude de sociologie de la déviance, Paris, Éditions A.M. Metailié, coll. «Observations », $247 p$.

BERTRAND, Marie-Andrée, avec la collaboration de Louise LANGELIERBIRON, Andrée BERTRAND-FAGNAN, Concetta DI PISA et Julia MCLEAN, (1998). Prisons pour femmes, Montréal, Éditions du Méridien, $400 \mathrm{p}$.

BESSIS, Sophie (2001). L'Occident et les autres. Histoire d'une suprématie, Paris, Éditions La Découverte, coll. «Cahiers libres », 340 p.

BEVERLEY, John (1999). Subalternity and Representation: Arguments in Cultural Theory, Durham, N.C. / London, Duke University Press, 202 p. versus McWorld, Paris, Desclée de 
BHALLA, A.S. et Frédéric LAPEYRE (1999). Poverty and Exclusion in a Global World, Basingstoke, Macmillan, $237 \mathrm{p}$.

BIHR, Alain et Rolland PFEFFERKORN (1999). Déchiffrer les inégalités, Paris, Syros / La Découverte, coll. "Alternatives économiques $», 576 \mathrm{p}$.

BOURDIEU, Pierre (1993). La misère du monde, Paris, Éditions du Seuil, coll. «Libre Examen », 949 p.

BOYER, Jean-Claude (1999). Amsterdam. La plus petite des grandes métropoles, Paris, L'Harmattan, 215 p.

CASTEL, Robert (1994). « La dynamique des processus de marginalisation: de la vulnérabilité à la désaffection ", Cahiers de recherche sociologique, vol. 22, p. 11-27.

CENTRE NATIONAL DE PRÉVENTION DU CRIME (2000). Cadre stratégique concernant la sécurité personnelle des femmes et des jeunes filles, Stratégie nationale sur la sécurité communautaire et la prévention du crime, Ottawa, Centre national de prévention du crime, $22 \mathrm{p}$.

COLLARD-GAMBIEZ, Michel et Colette COLLARD-GAMBIEZ (1998). Quand l'exclu devient l'élu. Vie partagée avec les sans-abri, Paris, Fayard, coll. "Les Enfants du fleuve », $434 \mathrm{p}$.

CONROY, John (2000). Unspeakable Acts, Ordinary People. The Dynamics of Torture, New York, Alfred A. Knopf, $304 \mathrm{p}$.

CORIN, Ellen (1986). "Centralité des marges et dynamique des centres", Anthropologie et Sociétés, vol. 10, $n^{\circ} 2$, p. 1-21.

COUTANT, Irène (2000). Politiques du squat. Scènes de la vie d'un quartier populaire, Paris, La Dispute, $222 \mathrm{p}$.

DAMON, Julien (1998). Vagabondage et mendicité : un exposé pour comprendre, un essai pour réfléchir, Paris, Éditions Flammarion, coll. «Dominos», $127 \mathrm{p}$.

DECLERCK, P. (2001). Les Naufragés. Avec les clochards de Paris, Paris, Plon, coll. "Terre humaine ", $454 \mathrm{p}$.

DIÈNE, Doudou (1998). La chaîne et le lien. Une vision de la traite négrière, Éditions UNESCO, 591 p.

DOGAN, Mattei et Robert PAHRE (1991). L'innovation dans les sciences sociales: la marginalité créatrice, Paris, Presses universitaires de France, coll. "Sociologies", 328 p.

DORAIS, Michel (1999). Éloge de la diversité sexuelle, Montréal, VLB Éditeur, $168 \mathrm{p}$.

DUCLOS, Denis (1998). Le complexe du loup-garou, Paris, Pocket, coll. "Agora », $272 \mathrm{p}$.

DUMONT, Fernand, Simon LANGLOIS et Yves MARTIN (1994). Traité des problèmes sociaux, Québec, Institut québécois de recherche sur la culture, $1127 \mathrm{p}$.

ELBAZ, M., A. FORTIN et G. LAFOREST (dir.) (1996). Les frontières de l'identité. Modernité et postmodernisme au Québec, Québec, Les Presses de I'Université Laval, 374 p.

EHRENREICH, Barbara (2001). Nickel and Dimed: On (Not) Getting By in America, New York, Metropolitan Books, $256 \mathrm{p}$.

FERGUSON, Marilyn (1995). Les enfants du Verseau, Paris, Éditions, coll. « J'ai lu », 440 p.

FOUCAULT, Michel (1975). Surveiller et punir, Paris, Gallimard, coll. « Tel», $318 \mathrm{p}$.

FOURNIER, Valérie (1999). Les nouvelles tribus urbaines: Voyage au cœur de quelques formes contemporaines de marginalité culturelle, Genève, Georg, $141 \mathrm{p}$.

FREITAG, Michel et Éric PINEAULT (1999). Le monde enchaîné, Québec, Nota bene, $331 \mathrm{p}$.

GAUTHIER, Madeleine (1994). « Entre l'excentricité et l'exclusion : les marges comme révélateur de la société", Sociologie et Sociétés, vol. XXVI, $\mathrm{n}^{\circ} 2$ (automne), p. 177-188.

GÉLINAS, Jacques B. (2000). La globalisation du monde. Laisser faire ou faire ?, Montréal, Écosociété, 340 p.

GREFFARD, Madeleine (2001). Portes ouvertes à l'École de la Rue, Montréal, Boréal, 209 p.

HARDT, Michael et Antonio NEGRI (2000). Empire, Paris, Éditions Exils, coll. « Essais », 559 p.

HEILBRUN, Carolyn G. (1997). Toward a Recognition of Androgyny, Replica Books.

HILT, C. (1998). "Après la reconnaissance, une nouvelle mise en marge ? Le mouvement des femmes et la structure des opportunités politiques au Canada, 1990-1997 ", Les Cahiers de recherche du GREMF (Groupe de recherche multidisciplinaire féministe), Québec, Université Laval, 66 p. HOOD, Suzanne, Berry MAYALL et Sandy OLIVER (dir.) (1999). Critical Issues in Social Research : Power and Prejudice, Buckingham, Open University Press, $177 \mathrm{p}$.

LABERGE, Danielle (dir.), avec la collaboration de membres du Collectif de recherche sur l'itinérance, la pauvreté et l'exclusion sociale (2000). L'errance urbaine, Montréal, Éditions MultiMondes, $439 \mathrm{p}$.

LABERGE, Danielle, Pierre LANDREVILLE et Daphné MORIN (2000). « Pratiques de déjudiciarisation de la maladie mentale: le modèle de l'urgence psychosociale - justice ", Criminologie, vol. 33, n², p. 81-107.
LAMBERT, Christophe (1999). Derrière les barreaux, Paris, Michalon, coll. «Droit de citer », 170 p.

LAMCHICHI, Abderrahim (1998). L'islamisme en question(s), Paris, Éditions L'Harmattan, coll. " Histoire et Perspectives méridiennes », $256 \mathrm{p}$.

LAMOUREUX, Diane (1998). Les limites de l'identité sexuelle, Québec, Éditions du Remue-ménage, $198 \mathrm{p}$.

LANGUEPIN Olivier (2000). Cuba : la faillite d'une utopie, Paris, Gallimard, coll. « Folio Actuel », 304 p.

LAVIGNE, Yves (1999). Death Dealers. Cocaine from Columbia, Heroin from Asia, Crack Cocaine from the Kid Next Door: The True Story of How Drugs Hit the Streets and Who Pays the Price, Toronto, HarperCollins, coll. « Paperback », $472 \mathrm{p}$.

LAVIGNE, Yves (1999). The Hell's Angels at War, Toronto, HarperCollins, coll. «English Pocket », 624 p.

LE MONDE DIPLOMATIQUE (2001). Peurs et menaces nouvelles, série $d$ 'articles portant sur les nouveaux désordres, les nouvelles insécurités, les nouvelles criminalités et les nouvelles manipulations, "Manière de voir 59 ", septembre-octobre, 98 p.

LESAGE, Marc (1997). Microcité, (ville), Montréal, Fidès, 243 p.

LESAGE, Marc (1986). Les vagabonds du rêve. Vers une société de marginaux?, Montréal, Les Éditions du Boréal, $169 \mathrm{p}$.

LIPIETZ, Alain (1998). La société en sablier, Paris, Éditions La Découverte et Syros, $386 \mathrm{p}$.

MACKENZIE, Edouardo et Alain DELPIROU (2001). Les cartels criminels, Paris, Presses universitaires de France, coll. « Criminalité internationale », $192 p$.

MEAGHAN, Diane E. (1999). Women in Black: The Political Economy of Prostitution in Cuba Under Market Socialism, communication présentée au Congrès de la Canadian Sociology and Anthropology Association of the Social Sciences and Humanities, University de Sherbrooke/Bishop University, Québec, 6-9 juin.

MEAGHAN, Diane E. (1999). "About face: The social construction of homelessness" dans Toronto the Good, communication présentée au Congrès de la Canadian Sociology and Anthropology Association of the Social Sciences and Humanities, University de Sherbrooke/Bishop University, Québec, $1^{\mathrm{er}}-4$ juin. Cette communication sera publiée chez Garammond Press, à Toronto (sous presse).

MEAGHAN, Diane E. (1991). "The political economy of sex-trade work: The social construction of stripping ",
The Journal of Social Problems, vol. 1, $n^{\circ} 1$, p. 6-24.

NATTIEZ, Jean-Jacques (1997). Wagner Androgyne (trad. de Stewart Spencer), Princeton, N.J., Princeton University Press, $380 \mathrm{p}$.

PARAZELLI, Michel, R. MORIN et G. MERCIER (1999). « La ville et le choc des imaginaires: populations marginalisées et revitalisation urbaine ${ }$, dans Lucie-K. MORISSET, Luc NOPPEN et Denis SAINT-JACQUES (dir.), Ville imaginaire, ville identitaire. Échos de Québec, Sainte-Foy, Nota bene, p. 209-227.

PAUGAM, Serge (2000). La disqualification sociale : essai sur la nouvelle pauvreté, Paris, Presses universitaires de France, coll. «Quadrige », 272 p.

PAUGAM, Serge (1996). L'exclusion: l'état des savoirs, Paris, Éditions La Découverte, $583 \mathrm{p}$.

PINÇON, Michel et Monique CHARLOTPINÇON (1997). Voyage en grande bourgeoisie. Journal d'enquête, Paris, Presses universitaires de France, $184 \mathrm{p}$. PINÇON, Michel et Monique CHARLOTPINÇON (1996). Grandes Fortunes. Dynasties familiales et formes de richesse en France, Paris, Payot, 375 p.

RACINE, Guylaine (2000). La production de savoirs d'expérience chez les intervenants sociaux. Le rapport entre l'expérience individuelle et collective, Montréal, L'Harmattan, coll. «Action et Savoir », 169 p.

ROBINSON, Gertrude J. (1998). « Monopolies of knowledge in Canadian communication studies: The case of feminist approaches", Canadian Journal of Communication, vol. 23, $\mathrm{n}^{\circ} 1$.

SANTE MENTALE AU QUÉBEC (2000). «Dossier Itinérance », vol. XXV, $\mathrm{n}^{\circ} 2$ (automne), $226 \mathrm{p}$.

SIBLEY, David (1995). Geographies of Exclusion: Society and Difference in the West, Londres, Routledge, 206 p.

SIMPSON, Anne (2001). Abus de confiance: les enfants et les jeunes de l'industrie du sexe, Ottawa, Caledon Institute of Social Policy (document disponible sur Internet).

SIOUI, Georges E. (1999). Pour une histoire amérindienne de l'Amérique, Québec, Presses de l'Université Laval, $160 \mathrm{p}$.

THÉRIAULT, LuC (2001). Social Housing with Community Support: A Study of the FOHM Experience, Ottawa, Caledon Institute of Social Policy, 31 p. (document disponible sur Internet).

TRÉPANIER, Jean (1999). « La justice des mineurs au Canada. Remises en question à la fin d'un siècle ", Criminologie, vol. $32, n^{\circ} 2$, p. 7-35. 
TRIGGER, Bruce G. (1976). The Children of Aataentsic, Montréal, McGill-Queen's University Press.

TSING, Anna Lowenhaupt (1993). In the Realm of the Diamond Queen: Marginality in an Out-of-the-Way Place, Princeton, NJ, Princeton University Press, $368 \mathrm{p}$.

UNESCO (2000). « Changer le regard. Élimination de la pauvreté en milieu urbain ». Rapport de la Commission programmatique mixte Pauvreté, $192 \mathrm{p}$.

VAN GENNEP, Arnold (1981). Les rites de passage, Paris, A. \& J. Picard.

WACQUANT, L. (1996). "The rise of advanced marginality: Notes on its nature and implications", Acta Sociologica, vol. 39, p. 121-139.

WOON, Yuen-Fong (1998). The Excluded Wife, Montreal et Ithaca, McGill-Queen's University Press, $320 p$.

\section{ETHNICITÉ / PLURIETHNICITÉ / IMMIGRATION / RÉFUGIÉS}

BALS, Myriam (1999). Les domestiques étrangères au Canada. Esclaves de l'espoir, Paris, L'Harmattan, coll. "Logiques sociales», $240 \mathrm{p}$.

BERNATCHEZ, S. et G. BOURGEAULT (1999). "La prise en compte de la diversité religieuse et culturelle à l'école et l'obligation d'accommodement. Aperçu des législations et jurisprudences au Canada, aux ÉtatsUnis, en France, au Royaume-Uni ", Études ethniques au Canada/ Canadian Ethnic Studies, vol. 31, n 1, p. 159-171.

BERNATCHEZ, S., F. GAGNON et M. PAGÉ (1989). "La Citoyenneté au Canada", dans F. GAGNON et M. PAGÉ (dir.), Cadre conceptuel d'analyse de la citoyenneté dans les démocraties libérales, Ottawa, Ministère du Patrimoine canadien, Recherche et analyse stratégiques, vol. 2, p. 92118.

BROUWER, Andrew (1999). Immigrants Need Not Apply, Ottawa, Caledon Institute of Social Policy, $18 \mathrm{p}$. (document disponible sur Internet).

DEMORGON, Jacques (2000). L'interculturation du monde, Paris, Economica, coll. «Anthropos », 178 p.

ELBAZ, Mikhaël (1993). " La communauté juive au Canada", dans S.A. GOLDBERG (dir.), Dictionnaire encyclopédique du judaïsme, Paris, Le Cerf, p. 1424-1432.

ELBAZ, Mikhaël (1990). « Figures de I'identité et de l'altérité: les Juifs dans le système urbain et ethnique montréalais", dans Ida SIMONBAROUH et Pierre-Jean SIMON, Les Étrangers dans la ville, Paris, L'Harmattan, p. 324-339.
FORTIN, Sylvie (2000). Destins et défis: la migration libanaise à Montréal, Montréal, Éditions Saint-Martin, $127 \mathrm{p}$.

GAGNON, J.E. et A. GERMAIN (1999). " The construction of a cosmopolitan city as an urban experience: The case of Montreal ", dans Louise NYSTROM et Colin FUDGE (dir.), City and Culture. Cultural Processes and Urban Sustainability, Karlskrona, The Swedish Urban Environment Council, p. 148-165.

GERMAIN, A., et D. ROSE (2000). Montreal. The Quest for a Metropolis, Chichester, John Wiley \& Sons, $306 \mathrm{p}$.

HELLY, D. et M. ELBAZ (dir.) (2000). Mondialisation, citoyenneté et multiculturalisme, Québec et Paris, Les Presses de I'Université Laval et L'Harmattan, $260 \mathrm{p}$.

JACOB, André et Joseph J. LEVY, en collaboration avec Jocelyne BERTHOT, Hector POBLETTE et Sonia SAUVÉ (1994). « Du départ forcé à l'intégration manquée ", dans F.-R. OUELLETTE et C. BARITEAU, Entre tradition et universalisme, Québec, Institut québécois de recherche sur la culture, p. $171-180$

JUTEAU, Danielle (2000). « Le défi de I'option pluraliste ", dans M. VENNE (dir.), La nation, Montréal, Québec/ Amérique, p. 199-214.

JUTEAU, Danielle (1999). L'ethnicité et ses frontières, Montréal, Les Presses de l'Université de Montréal, 226 p.

JUTEAU, Danielle (1999). « Rapports ethniques au Canada: recherche et politique", dans Ida SIMONBAROUH (dir.), Migrations internationales et relations inter-ethniques - Recherche, politique et sociétés, Paris, L'Harmattan.

LAM VAN BE (1995). L'immigration et les communautés culturelles du Québec, 1968-1995: bibliographie sélective annotée, Saint-Laurent, Éditions Sans Frontières, $198 \mathrm{p}$.

LAPERRIERE, Anne, Varpu LINDSTRÖM et Tamara PALMER SEILER (dir.) (1996). Immigration and Ethnicity in Canada / Immigration et ethnicité au Canada, Montréal, Association for Canadian Studies/Association d'études canadiennes, $214 \mathrm{p}$.

LEY, D. et A. GERMAIN (2000). « The new immigrant face of Canadian cities? ", Plan Canada, vol. 40, n² p. 29-32.

McALL, Christopher (1995). "Les murs de la cité : territoires d'exclusion et espaces de citoyenneté ", Lien social et Politiques - RIAC, $\mathrm{n}^{\circ} 34$, p. 81-92.

MEINTEL, D. et J. LEGAL (1995). Les jeunes d'origine immigrée: Rapports familiaux et les transitions de vie. Le cas des jeunes chiliens, grecs, portugais, salvadoriens et vietnamiens, Québec, Ministère des Affaires internationales, de I'Immigration et des Communautés culturelles, coll. «Études et Recherches ", $n^{\circ} 10,133 p$.

ROUSSEAU, Cécile (2000). « Les réfugiés à notre porte : violence organisée et souffrance sociale ", Criminologie, vol. 33, n 1, p. 185-201.

SAYAD, Abelmalek (1999). La double absence. Des illusions de l'émigré aux souffrances de l'immigré, Préface de Pierre Bourdieu, Paris, Liber, Seuil, $437 \mathrm{p}$.

STOICIU, Gina (1996). «L'intégration, un construit théorique ", dans K. FALL, R. HADJ-MOUSSA et D. SIMEONI, Les convergences culturelles dans les sociétés pluriethniques, Sainte-Foy, Presses de I'Université du Québec, p. 15-30.

SYMONS, G. (1999). «The racialization of the street gang issue in Montreal: A police perspective", Canadian Ethnic Studies / Études ethniques au Canada, vol. XXXI, n 2, p. 1-15.

TROPER, H. et M. WEINFELD (dir.) (1999). Ethnicity and Public Policy: Case Studies in Canadian Diversity, Toronto, University of Toronto Press. WEINFELD, M. (1999). "The integration of Jewish immigrants in Montreal : Models and dilemmas of ethnic match", dans Daniel J. ELAZAR et Morton WEINFELD (dir.), Still Moving, New Brunswick, NJ, Transaction Publications.

WIEVIORKA, Michel (2000). La Différence, Paris, Balland, coll. "Voix et Regards », $200 \mathrm{p}$.

\section{EXCLUSION - INCLUSION}

ACTION SOCIALE, (2000). L'exclusion. Définir pour en finir, Paris, Dunod, $220 \mathrm{p}$.

AUDOLLENT, D.G. et D. FAYARD (1999). Combattre l'exclusion, Toulouse, Éditions Milan, coll. «Les essentiels Milan », $64 \mathrm{p}$.

BERTRAND, Marie-Andrée, assistée de Valérie Demers et al. (1998). Les obstacles au changement dans la condition des femmes, Rapport de l'étude portant sur les cheminements de carrière de vingt-deux femmes cadres et professionnelles travaillant dans le champ sociopénal, Montréal, Centre international de criminologie comparée, $51 \mathrm{p}$.

CAHIERS DE RECHERCHE SOCIOLOGIQUE (1994). « Marginalité et exclusion sociales ", Cahier $n^{\circ} 22,158 \mathrm{p}$.

CASTEL, Robert (1995). " Les pièges de l'exclusion", Lien social et Politiques-RIAC, $\mathrm{n}^{\circ} 34, \mathrm{p} .13-23$.

De RIVOYRE, Frédéric (dir.) (2000). Dire l'exclusion, Toulouse, Eres, 158 p.
DOURA, Fred (1999). Mondialisation: exclusion sociale et marginalisation des pays sous-développés, Montréal, CIDIHCA, $254 \mathrm{p}$.

DUPUIS, Renée (2001). Quel Canada pour les Autochtones? La fin de l'exclusion, Montréal, Éditions du Boréal, $176 \mathrm{p}$.

ELIAS, Norbert, John L. SCOTSON (1997). Logiques de l'exclusion. Enquête sociologique au cœur des problèmes d'une communauté, Paris, Fayard, $278 \mathrm{p}$.

FRETIGNE, Cédric (1999). Sociologie de l'exclusion, Paris, L'Harmattan, coll. "Logiques sociales », 208 p.

GARNIER-MULLER, Anne (2000). Les inutiles. Survivre au quotidien en banlieue et dans la rue, Paris, Les Éditions de l'Atelier, 240 p.

GUYENNOT, Claudel (1998). Insertion: un problème social, Montréal, L'Harmattan, coll. "Logiques sociales", $254 \mathrm{p}$.

KRAKOVITCH, Odile et SELLIER, Geneviève (dir.) (2001). L'Exclusion des femmes: masculinité et politique dans la culture au XXe siècle, Bruxelles Complexe, $220 \mathrm{p}$.

KRAKOVITCH, Odile, SELLIER, Geneviève et VIENNOT, Eliane (dir.) (2001). Femmes de pouvoir, mythes et fantasmes, Paris, L'Harmattan, 238 p. KRESS, Susan (1997). Carolyn G. Heilbrun, Feminist in a Tenured Position, Charlottesville, VA, University Press of Virginia, $288 \mathrm{p}$.

LABERGE, Danielle et Shirley ROY (1994). "Marginalité et exclusions sociales", Cahiers de recherche sociologique, $n^{\circ} 22$, p. 5-10.

MAISONDIEU, Jean (1997). Fabrique des exclus, Paris, Bayard-Presse, coll. "Société ", 264 p.

PIZANIAS, C. et S. FRIDERES (1999). Freedom Within the Margins. The Politics of Exclusion, Calgary, University of Calgary, $310 \mathrm{p}$.

SOULET, Marc-Henry (1998). «L'exclusion: usages et mésusages d'un concept", Revue suisse de sociologie, vol. 24, n³, p. 431-458.

STRONG-BOAG, Veronica, Sherrill E. GRACE, Avigail EISENBERG, Gabriele HELMS, Matt JAMES et Paddy RODNEY (dir.) (1998). Painting the Maple: Essays on Race, Gender, and the Construction of Canada, Vancouver, University of British Columbia Press, $290 \mathrm{p}$.

THOMAS, Hélène, (1997). Production des exclus, Paris, Presses universitaires de France, coll. "Sociologie d'aujourd'hui ", $214 \mathrm{p}$.

TYMCHUK, Alexander J., Charlie LAKIN et Ruth LUCKASSON (dir.) (2001). The Forgotten Generation. 
The Status of Adults with Mild Cognitive Limitations, Baltimore, Paul H. Brookes Publishing Co., $416 \mathrm{p}$.

XIBERRAS, Martine (2000). Les théories de l'exclusion, Paris, Armand Colin, $242 \mathrm{p}$

" FOLIE » - « SANTÉ MENTALE » PSYCHOTROPES / ALTERNATIVE

CANGUILHEM, Georges (1966). Le normal et le pathologique, Paris, Presses universitaires de France, 1966. Voir aussi la deuxième édition parue en 1988, Paris, Presses universitaires de France, coll. "Quadrige».

CHAUMON, Franck et Catherine MACHET (dir.) (1999). Inactualité de la folie, Montréal, L'Harmattan, coll. "Pratiques de la Folie », 218 p.

COHEN, David et Suzanne CAILLOUXCOHEN (1995). Guide critique des médicaments de l'âme, Montréal, Éditions de l'Homme, $414 \mathrm{p}$.

DANJOU, Marie-Noëlle (2001). Raison et folie, Paris, L'Harmattan, coll. "Psychanalyse et Civilisation».

DE SIVRY, Sophie, Philippe MEYER et Laurent BECCARIA (1998). L'art et la folie, Le Plessis-Robinson, Essonne, Institut d'Édition Sanofy-Synthelabo, coll. "Les empêcheurs de penser en rond $", 128 \mathrm{p}$.

FOUCAULT, Michel, Les anormaux: cours au Collège de France (19741975), édition établie en 1999 sous la direction de F. EWALD, A. FONTANA, V. MARCHETTI et A. SALOMONI, Paris, Gallimard, 351 p.

FOUCAULT, Michel (1995). Maladie mentale et psychologie, Paris, Presses universitaires de France, coll. "Quadrige », $112 \mathrm{p}$.

FOUCAULT, Michel (1973). Moi, Pierre Rivière, ayant égorgé ma mère, ma sœur et mon frère, Paris, Gallimard, coll. « Archives », 349 p.

FOUCAULT, Michel (1972). Histoire de la folie à l'âge classique suivi de Mon corps, ce papier, ce feu et La folie, I'absence d'œuvre, Paris, Gallimard, coll. "Bibliothèque des histoires », 613 p.

LATOUR Bruno (1996). Petite réflexion sur le culte moderne des dieux fétiches, Éditions Synthelabo, Le Plessis, coll. "Les empêcheurs de penser en rond ", $103 \mathrm{p}$.

LEMIEUX, Roger R. (1995). Accueillir la folie, Piémont, Noir sur Blanc, $334 \mathrm{p}$.

LOPEZ, Gérard (2000). Le vampirisme au quotidien, La Ferté-Saint-Aubin, Loiret, Atelier de L'Archer, coll. "Questions de temps », 104 p.

RIGOLI, Juan (2001). Lire le délire, Paris, Fayard, $500 p$.
SOLOMON, Andrew (2001). The Noonday Demon. An Atlas of Depression, New York, Scribner, 571 p.

THUILLIER, Jean (1996). La folie. Histoire et dictionnaire, Paris, Robert Laffont, coll. «Bouquins », 840 p.

ZARIFIAN, Edouard (2001a). Une certaine idée de la folie, La Tour-d'Aigues, Vaucluse, Éditions Aube de I'Intervention, $96 \mathrm{p}$.

ZARIFIAN, Edouard (2001b). La force de guérir, Paris, Odile Jacob, $180 \mathrm{p}$.

ZARIFIAN, Edouard (2000). Les jardiniers de la folie, Paris, Odile Jacob, $295 \mathrm{p}$.

ZARIFIAN, Edouard (1998). Des paradis plein la tête, Paris, Odile Jacob, coll. «Opus», 214 p.

\section{SCIENCES RELIGIEUSES}

DAVIS, Nathalie Zémon (1997). Juive, catholique, protestante: trois femmes en marge au XVIIe siècle, Traduit de Women on the Margins, à partir des biographies de Marie de I'Incarnation, Maria Sibylla Merian et Gleuckel d'Hamelin, Paris, Éditions du Seuil, coll. « La Librairie du XXe siècle », 389 p.

JENKINS, Philip (2000). Mystics and Messiahs: Cults and New Religions in American History, Oxford, Oxford University Press, $294 \mathrm{p}$.

REMOND, René et Marc LEBOUCHER (2000). Le Christianisme en accusation, Paris, Desclée de Brouwer, 159 p.

\section{ART, CINÉMA ET MARGES}

BEUGNET, Martine (2001). Marginalité, sexualité, contrôle dans le cinéma français contemporain, Paris, L'Harmattan. 